\title{
V2X-Communication Assisted Interference Minimization for Automotive Radars
}

\author{
Jingxuan Huang ${ }^{1,2}$, Zesong Fei ${ }^{1,2, *}$, Tianxiong Wang ${ }^{1,2}$, Xinyi Wang ${ }^{1,2}$, Fan Liu ${ }^{3}$, Haijun Zhou ${ }^{1,2}$, \\ J. Andrew Zhang ${ }^{4}$, Guohua Wei ${ }^{1}$ \\ ${ }^{1}$ School of Information and Electronics, Beijing Institute of Technology, Beijing 100081, China \\ ${ }^{2}$ State Key Laboratory of Wireless Mobile Communications, China Academy of Telecommunications Technology (CATT) \\ ${ }^{3}$ Department of Electronic and Electrical Engineering, University College London, London WC1E 7JE, U.K. \\ ${ }^{4}$ University of Technology Sydney, Sydney, NSW 2007, Australia \\ ${ }^{*}$ The corresponding author, email: feizesong@bit.edu.cn
}

\begin{abstract}
With the development of automated driving vehicles, more and more vehicles will be fitted with more than one automotive radars, and the radar mutual interference will become very significant. Vehicle to everything (V2X) communication is a potential way for coordinating automotive radars and reduce the mutual interference. In this paper, we analyze the positional relation of the two radars that interfere with each other, and evaluate the mutual interference for different types of automotive radars based on Poisson point process (PPP). We also propose a centralized framework and the corresponding algorithm, which relies on V2X communication systems to allocate the spectrum resources for automotive radars to minimize the interference. The minimum spectrum resources required for zero-interference are analyzed for different cases. Simulation results validate the analysis and show that the proposed framework can achieve near-zero-interference with the minimum spectrum resources.
\end{abstract}

Key words: Automotive radars, V2X communications, radar interference, spectrum allocation.

\section{INTRODUCTION}

Automated driving vehicles (ADVs) are becoming a reality with the advancing of artificial intelligence and electronic technologies. Recently, advanced driver assistant systems (ADASs) have already been commercialized, while fully automated vehicles have been validated successfully in laboratory. By 2015, $15 \%$ of vehicles were equipped with ADASs, while around $50-60 \%$ of vehicles are expected to have higher-level automation by 2020 [1]. With the help of $\mathrm{ADV}$, it is possible to prevent more than 85 percent of traffic accidents caused by human errors [2]. Traffic jam will also be reduced by the coordination among ADVs.

Automotive radar is widely used in driver assisted systems to enable safety features such as adaptive cruise control and automatic emergency braking. Compared with camera and LIDAR, automotive radar is advantageous in that it can work at any time and under all weather conditions. On the other hand, vehicle to everything (V2X) communication is also a key component for ADVs [3]. By connecting to the cloud and the fog using V2X systems, each individual vehicle can get global information, unlimited computing and storage resources and cooperation abilities.

Therefore it is favorable to have both sensing and communication capabilities equipped on the ADV, where the co-existing and the co-designed radar and communication systems are typically required [4]. Some schemes were proposed to enable radar and communication systems sharing the same spectrum by eliminating the interference between these two systems [5]-[9], thus the frequency resources can be used more efficiently. The work in [5] proposed an opportunistic spectrum sharing scheme where the communication system utilizes the space and frequency spectra when they are not occupied by radar. The authors in [6] proposed a null-space projection method, in which radar signal is projected onto the null-space of the interference channels between the radar and the communication base station (BS). In [7], [8], the authors considered spectrum sharing between MIMO radar and multi-user MIMO (MU-MIMO) communications. The authors in [9] proposed an adaptive signal processing method for communication system to remove the interference from radar.

As a step further, some researchers focus on realizing radar and communication functionalities by use of shared waveforms [10]-[13]. In [10], the authors proposed to exploit IEEE 802.11ad protocol for target detection. In [11], [12], sensing and communication systems were integrated on a C-RAN based mobile network. Recently, an MU-MIMO radar-communication waveform was designed to minimize the downlink multi-user interference while formulating a desired radar transmit beampattern [13]. In [14], the authors investigated how to use radar signal for MIMO communication beam training. Nevertheless, it still remains an open issue that how 
the radar system can benefit from the communication systems.

The ADVs may be a suitable scenario that communication system can benefit to radar. With the increasing number of ADVs, the number of automotive radars will also increase. On the other hand, the spectrum resource for automotive radar is limited, thus enormous number of radars have to share the same spectrum, and significant radar interference will arise due to the lack of coordination among them. Particularly, interfering radars that are implemented in vehicles traveling in the opposite direction and work in the collision frequency band will create ghost targets that cause false alarm and noise-like interference which degrades the target detection performance [15], [16]. The works in [17] and [18] suggested an approach that randomizes chirp sweeping frequency to reduce the false alarm probability. The authors in [19] modeled the automotive radar interference by using stochastic geometry, and proposed an optimization methodology to calculate the optimum random access probability that can reduce interference. In addition to the aforementioned techniques, V2X communication can introduce coordination between automotive radars and further reduce the interference, i.e., bring gain for radars.

In this paper, we propose a new centralized framework that utilizes V2X communication system to allocate spectrum resources for the automotive radars and reduce interference. Similar to the conventional frequency division multiplexing (FDM) method, the frequency band is divided into several sub-bands. However, the number of sub-bands is limited, while the number of automotive radars is huge, it is impossible to allocate non-reused orthogonal band for each radar as mobile communication system. Thus in our proposed framework, the BS allocates the reused spectrum resources to minimize radar interference using a greedy algorithm based on the location and direction information of radars. We also analyze the positional relation of interfering radars, and evaluate the interference in different cases based on Poisson point process (PPP). Moreover, we analyze the minimum spectrum resources required for zero-interference. Simulation results show that our proposed framework can eliminate interference by using the minimum spectrum resources, while achieving higher radar detection probability compared with random spectrum access method when the spectrum resources are not sufficient.

The rest of this paper is organized as follows. In the second section, we formulate the model of radar interference. In the third section, we propose the spectrum allocation framework and the algorithm. In the fourth section, we analyze the average interference and the minimum spectrum resources required for completely avoiding interference. In the fifth section, we show the simulation results. At last we conclude the paper.

\section{SYSTEM MODEL}

In this section, we formulate the model of radar signal and radar interference. Then we construct the vehicle model that how radars are equipped on the vehicle. At last we present the positional relation of the two radars that interfere with each other.

\subsection{Signal model and interference model}

In practical scenarios, it is more likely for two radars to cause noise-like interference rather than ghost target [16], especially for frequency modulated continuous wave (FMCW) radars that are widely used in vehicles. Thus in this paper, we focus on noise-like interference, and utilize signal to interference plus noise ratio (SINR) to evaluate the performance of radar.

The power of the signal that is transmitted by the radar and reflected from the target can be characterized by the radar equation [20] as follows:

$$
S=\frac{P_{t} G_{t}}{4 \pi R^{2}} \times \frac{\sigma_{c}}{4 \pi R^{2}} A_{e},
$$

where $S$ is the received signal power, $P_{t}$ is the transmitting power, $G_{t}$ is the transmitter antenna gain, $R$ is the distance from the radar to the target, $\sigma_{c}$ is the radar cross-section area (RCS) of the target, and

$$
A_{e}=\frac{G_{r} \lambda^{2}}{4 \pi}
$$

is the effective aperture of the receiver antenna, where $G_{r}$ is the receiver antenna gain, and $\lambda$ is the wavelength. Note that $S$ can be equivalently divided into three parts as

$$
S=\gamma_{t} \times \gamma_{r} \times A_{e},
$$

where

$$
\gamma_{t}=\frac{P_{t} G_{t}}{4 \pi R^{2}}
$$

represents the incident signal to the target, and

$$
\gamma_{r}=\frac{\sigma_{c}}{4 \pi R^{2}}
$$

denotes the reflection from the target. Thus if the transmitted signal is received by an opposing radar that works in the same spectrum, the interference power will only depend on $\gamma_{t}$ and $A_{e}$, and the interference power from radar $m$ to radar $n$ can be expressed as 


$$
\begin{aligned}
I_{m n} & =\frac{P_{t m} G_{t m}}{4 \pi R_{m n}{ }^{2}} \times \frac{G_{r n} \lambda^{2}}{4 \pi} \\
& =P_{t m} G_{t m} G_{r n}\left(\frac{\lambda}{4 \pi}\right)^{2} R_{m n}{ }^{-2},
\end{aligned}
$$

where $P_{t m}$ and $G_{t m}$ are the transmitting power and transmitter antenna gain of radar $m$, respectively, $G_{r n}$ is the receiver antenna gain of radar $n$, and $R_{m n}$ is the distance between the two radars.

\subsection{Vehicle model}

Both automated driving vehicle and vehicle with ADASs could be fitted with more than one radar [21]. There will be a long range radar (LRR) on the front of the vehicle for sensing distances of $10-250 \mathrm{~m}$, enabling the features such as adaptive cruise control (ACC) and brake assist. There will also be several medium range radars (MRRs) and short range radars

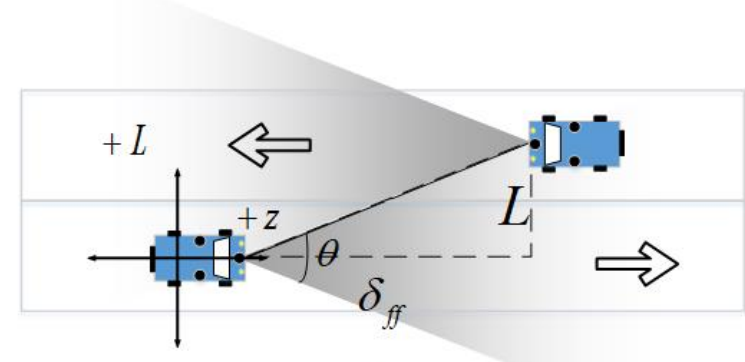

(a)

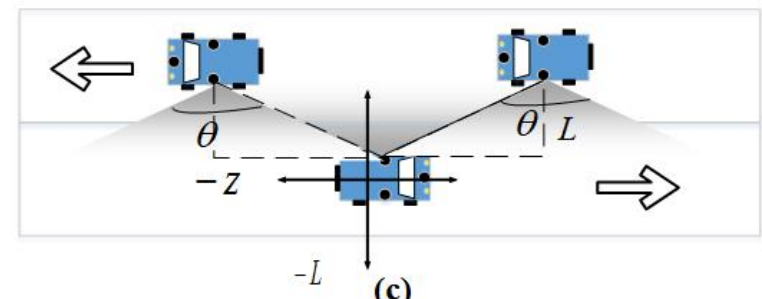

(c)

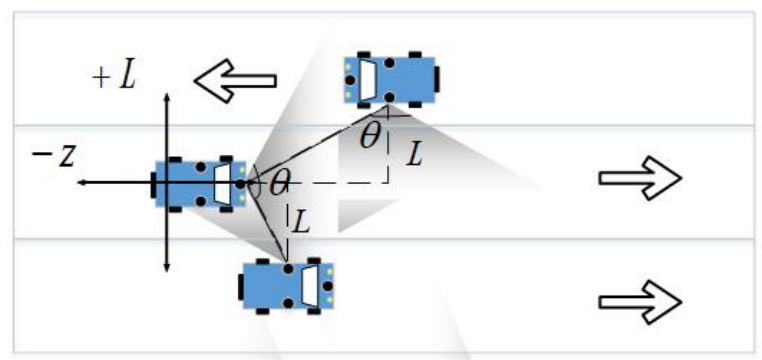

(b)

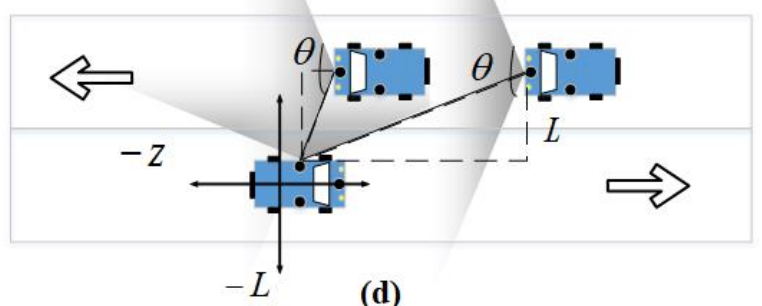

(d)

Fig. 1 A simple layout of interfering automotive radars. (a) Front radar to front radar. (b) Side radars to front radar. (c) Side radars to side radar. (d) Front radars to side radar.

(SRRs) on the front, side or the back of the vehicle for sensing distances of 1-100 $\mathrm{m}$ and 0.15-30 m, respectively. The MRRs/SRRs on the front and side are for collision warning, cross traffic alert and blind spot detection, while the MRRs/SRRs on the back mainly provide backup parking assist [22].

Since the radars on the back are mainly used for parking, the interference generated to the vehicles can be neglected in general. We therefore only consider radars on the front and the side. In this paper, we assume that each vehicle is fitted with three radars, i.e., one front radar, and two side radars on the left and right respectively. Each vehicle is equipped with V2X systems that work in the different frequency bands with the automotive radars. For simplicity, we assume each radar shares the same parameters, and denote the transmit power as $P_{t}$, the antenna gain as $G$, and the antenna beam width as $\theta$. Note that the three radars in the same car have different direction, thus the interference among them is negligible, and can be eliminated by self-cancellation.

\subsection{The geometrical layout of radar interference}

A geometrical layout of interfering automotive radars is illustrated in Fig. 1, which shows the potential interference between two opposite radars on the road. In Fig. 1, we decompose the distance into two directions, one is the extension direction of roads, denoted as $\pm z$, and the other is the vertical direction, denoted as $\pm L$, thus the location of a vehicle can be expressed as $(\mathrm{z}, \mathrm{L})$. The interested vehicle located on the zero point is called the typical vehicle, and the driving direction of the typical vehicle is $+z$.

First we will investigate the interference to the front radar of the typical vehicle. The front radar will be interfered by other front radars of the vehicles traveling in the opposite direction. Consider the antenna beam width and ignore sidelobes, the interfering front radars are mounted on the vehicle beyond a minimum distance, as shown in Fig. 1 (a). The location of the interfering front radar satisfies

$$
\left\{\begin{array}{c}
L>0 \\
z>\delta_{\text {ff }}=\frac{L}{\tan \frac{\theta}{2}},
\end{array}\right.
$$

where $\delta_{f f}$ is the minimum distance. If $\theta>\frac{\pi}{2}$, the front radar of the typical vehicle will also receive the 
interference from the side radars of the vehicles that locate between a minimum distance $\delta_{0 s f}$ and a maximum distance $\delta_{1 s f}$ on the other lanes, as shown in Fig. 1 (b). Specifically, the location of the interfering side radar satisfies

$$
\left\{\begin{array}{c}
L \neq 0 \\
\delta_{0 s f}<z<\delta_{1 s f}
\end{array},\right.
$$

where

$$
\begin{gathered}
\delta_{0 s f}=\frac{L}{\tan \frac{\theta}{2}}, \\
\delta_{1 s f}=L \tan \frac{\theta}{2} .
\end{gathered}
$$

Similarly, the side radar will be interfered by other opposite side radars equipped on the vehicles of other lanes, as shown in Fig. 1 (c). For example, for the left side radar of the typical vehicle, the location of the interfering side radar satisfies

$$
\left\{\begin{array}{c}
L>0 \\
-\delta_{s s}<z<\delta_{s s}
\end{array},\right.
$$

where

$$
\delta_{s s}=L \tan \frac{\theta}{2}
$$

And if $\theta>\frac{\pi}{2}$, this left side radar will be interfered by the front radar at the location of

$$
\left\{\begin{array}{c}
L>0 \\
\delta_{0 f s}<z<\delta_{1 f s}
\end{array},\right.
$$

where

$$
\begin{aligned}
& \delta_{0 f \mathrm{~s}}=\frac{L}{\tan \frac{\theta}{2},} \\
& \delta_{1 f \mathrm{~s}}=L \tan \frac{\theta}{2},
\end{aligned}
$$

as shown in Fig. 1 (d).

From the discussion above, we observe that the most significant interference is generated from one side radar to another. If the antenna beam width is narrow, the interference between a side radar and a front radar will disappear, and the interference between two front radars is small because the distance is quite large. However, it is always possible that two opposite side radars are closer to each other.

\section{THE PROPOSED INTERFERENCE MINIMIZATION FRAMEWORK AND ALGORITHM}

In this section, we introduce the framework that utilizes V2X communication network to allocate spectrum resources and minimize automotive radar interference. We formulate an optimization problem, which is then solved via a greedy algorithm.

\subsection{V2X Communication assisted radar spectrum resource allocation framework}

It is impossible to allocate non-reused orthogonal spectrum resources allocation for automotive radars. Typical automotive radar, e.g., FMCW radar, requires a large bandwidth of at least $200 \mathrm{MHz}$ [26]. Even though there is totally $2 \mathrm{GHz}$ bandwidth for automotive radars at $77 \mathrm{GHz}$ in most countries [16], only 10 non-reused orthogonal sub-bands can be used. Considering the huge amount of automotive radars, especially for dense traffic situation, it is meaningful to study how to allocate the limited resource based on location information to minimize the interference.

In the proposed framework, the vehicles in a section of road belong to the same base station. For vehicle $j$, denoting the index of its front radar, left side radar and right side radar as $3 j-2,3 j-1$ and $3 j$, respectively, we have

$$
M=3 \times J,
$$

where $M$ is the total number of automotive radars and $J$ is the total number of vehicles. The frequency band of automotive radar is divided into $T$ orthogonal sub-bands with the same bandwidth. Denote the sub-bands allocation matrix as $\boldsymbol{F} \in\{0,1\}^{M \times T}$, and denote the $m$ th row of $\boldsymbol{F}$ as $\boldsymbol{f}_{m}$. We have $\boldsymbol{f}_{\boldsymbol{m}} \in\{0,1\}^{1 \times T}$, where $\boldsymbol{f}_{\boldsymbol{m}}(\mathrm{t})=1$ means the $t$ th sub-band is allocated to radar $m$.

Each vehicle uploads its parameters to the base station, including the locations and the orientations for each radar. The base station can also require speed information to predict the location of vehicles during two uploading slots. After that, the base station calculates the potential interference between the automotive radars. The potential interference means there is interference if two radars were allocated with the same sub-band. Location, radar orientations and antenna beam widths are substituted to Eq. (7-12) to decide whether there exists potential interference between two radars. If two radars interfere with each other, the base station further calculates the distance between these two radars, and then gets a potential interference matrix $\boldsymbol{P} \in \mathbb{R}^{M \times M}$ based on Eq. (6), where

$$
\boldsymbol{P}(\mathrm{m}, \mathrm{n})=\left\{\begin{array}{l}
0, \text { no potential interference } \\
P_{t} G^{2}\left(\frac{\lambda}{4 \pi}\right)^{2} R_{m n}{ }^{-2}, \text { otherwise }
\end{array}\right.
$$

is the potential interference from radar $m$ to radar $n$. Note that $\boldsymbol{P}$ is a symmetric matrix, and main diagonal elements are zero. The base station needs to allocate sub-bands for radars to minimize the total interference. The optimization problem is given as 
follows:

$$
\begin{gathered}
\min _{\boldsymbol{F}} \operatorname{sum}(\boldsymbol{P} \cdot \boldsymbol{F}) \\
\text { s.t. } \boldsymbol{f}_{m} \in\{0,1\}^{1 \times \mathrm{T}}, \\
\left\|\boldsymbol{f}_{m}\right\|_{0}=1,
\end{gathered}
$$

where $\left\|\boldsymbol{f}_{m}\right\|_{0}=1$ means each radar can be allocated with only one sub-band.

\subsection{Interference minimization algorithm}

Here we propose a greedy algorithm to solve the minimization problem. First the sub-bands allocation matrix is initialized as a zero matrix. Then the base station allocates the sub-bands for radars in the order of their index. For each radar, the base station calculates the interference that will be introduced to all sub-bands, and allocates the radar with the sub-band that the introduced interference is minimum. The detailed algorithm can be found in Algorithm I, where $\boldsymbol{p}_{\boldsymbol{m}}$ is the $m$ th row of $\boldsymbol{P}$.

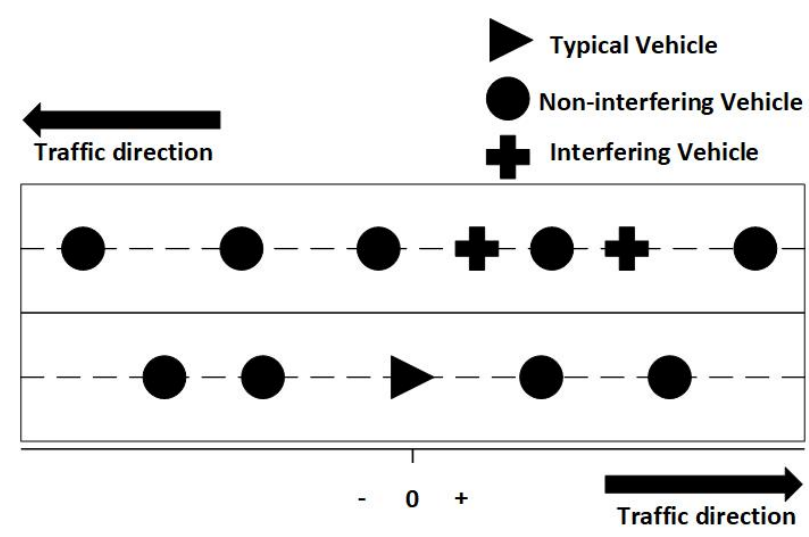

Fig. 2 The illustration of the proposed road model.

\section{THEORETICAL ANALYSIS OF RADAR INTERFERENCE}

In this section, we analyze the mean power of radar interference by using PPP. Moreover, we analyze the minimum number of sub-bands required for zero-interference.

\subsection{Radar interference mean}

Without loss of generality, we consider the two-lane road with two opposing traffic directions. For a certain lane, we assume there is no correlation between the locations of vehicles, and all vehicles are located in the centerline of the road. Under this assumption, the locations of vehicles resemble a unidimensional PPP in $\mathbb{R}^{1}$ with a homogeneous linear intensity $\rho$ [23]. For a typical vehicle, the interference to its front radar and left radar comes

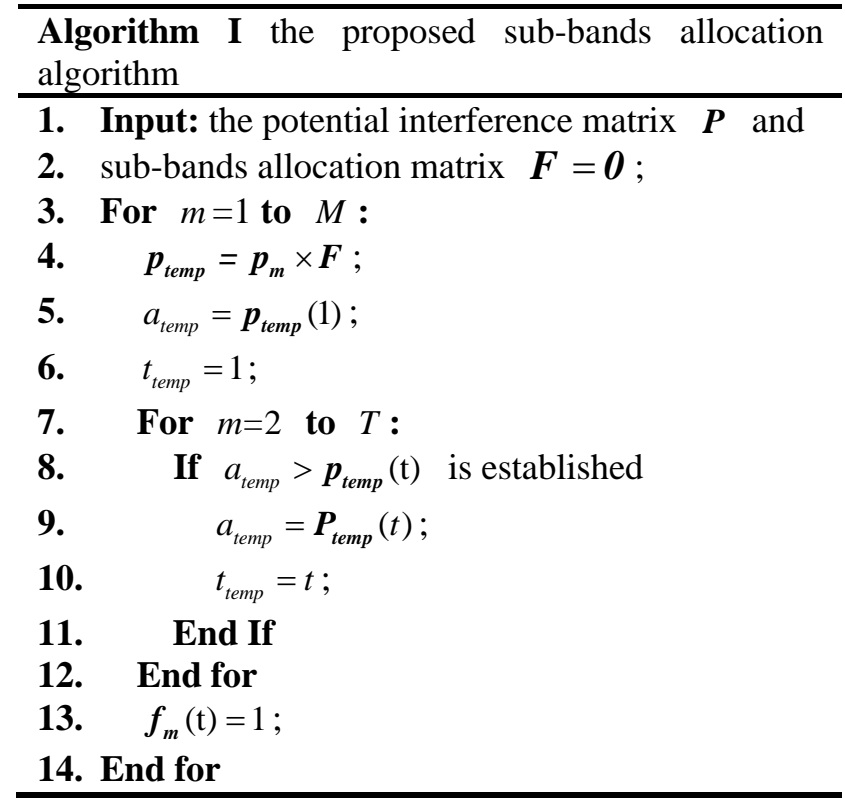

from the vehicles in the other lane. We denote the set of vehicles in the other lane as $\Phi$. Without the proposed framework, each radar accesses one of $T$ orthogonal sub-bands randomly, thus the probability that two opposing radar access the same sub-band and interfere with each other is $1 / T$. We apply random thinning on the vehicles set $\Phi$ with a retention probability $\xi=1 / T$. An illustration of the proposed road model is shown in Fig. 2.

Utilizing the result in Subsection 2.3 we can get the expressions of interferer set. For the front radar of the typical vehicle, the interferer front radars set can be expressed as

$$
\Theta_{f f}=\left\{z: z \in \Phi, M_{f f}(z)=1\right\},
$$

where $\mathrm{M}_{f f}(z)$ is a random mark defined as

$$
\mathrm{M}_{f f}(\mathrm{z})=\left\{\begin{array}{l}
0, \quad \mathrm{z} \leq \delta_{\text {ff }} \\
B(\xi), z>\delta_{f f}
\end{array},\right.
$$

and $B(\xi)$ is a Bernoulli random variable with selection probability $\xi$. For the interferer left radars set $\Theta_{s f}$, the random mark $M_{s f}(z)$ can be expressed as

$$
\mathrm{M}_{s f}(\mathrm{z})=\left\{\begin{array}{l}
B(\xi), \delta_{0 s f}<z<\delta_{1 s f} \\
0, \quad \text { otherwise }
\end{array} .\right.
$$

Similarly for the left radar of the typical car, the random mark for the interferer front radars set $\Theta_{f s}$ is

$$
\mathrm{M}_{f s}(z)=\left\{\begin{array}{l}
B(\xi), \delta_{0 f s}<z<\delta_{1 f s} \\
0, \quad \text { otherwise }
\end{array}\right.
$$

The random mark for the interferer left radars set $\Theta_{s s}$ is

$$
\mathrm{M}_{s \mathrm{~s}}(\mathrm{z})=\left\{\begin{array}{l}
B(\xi),-\delta_{s s}<z<\delta_{s s} \\
0, \quad \text { otherwise }
\end{array} .\right.
$$

For the typical vehicle at the zero point, the 
distance to another vehicle in the next lane can be expressed as

$$
R(\mathrm{z})=\left(\mathrm{L}_{1}^{2}+\mathrm{z}^{2}\right)^{\frac{1}{2}}
$$

where $L_{1}$ is the lane width. Neglecting the length and width of the vehicle, Eq. (22) can also express the distance between two radars, and the power of interference can be expressed as

$$
\begin{aligned}
I(\mathrm{z}) & =P_{t} G^{2}\left(\frac{\lambda}{4 \pi}\right)^{2} R(\mathrm{z})^{-2} \\
& =\gamma\left(\mathrm{L}_{1}+\mathrm{z}^{2}\right)^{-1},
\end{aligned}
$$

where

$$
\gamma=P_{t} G^{2}\left(\frac{\lambda}{4 \pi}\right)^{2} .
$$

Thus for the front radar of the typical car, the aggregated interference $P_{f}$ can be written as

$$
\begin{aligned}
P_{f} & =\sum_{z \in \Theta_{f f}} I(\mathrm{z})+\sum_{z \in \Theta_{s f}} I(\mathrm{z}) \\
& =\sum_{z \in \Theta_{f f}} \gamma\left(L_{1}^{2}+z^{2}\right)^{-1}+\sum_{z \in \Theta_{s f}} \gamma\left(L_{1}^{2}+z^{2}\right)^{-1} . \\
& ------~
\end{aligned}
$$

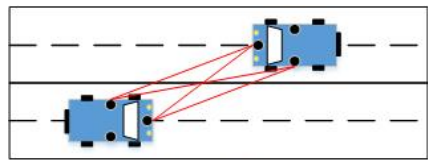

(a) Two lanes

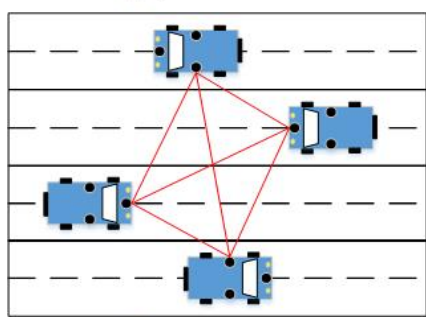

(b) Four lanes

Fig. 3 Full-interference set of the straight road.

By applying Campbell theorem [24] to Eq. (25), we can calculate the mean value of $P_{f}$ :

$$
\begin{aligned}
\overline{P_{f}} & =\mathbb{E}\left[P_{f}\right] \\
& =\mathbb{E}\left[\sum_{z \in \Theta_{f f}} \gamma\left(L_{1}^{2}+z^{2}\right)^{-1}\right]+\mathbb{E}\left[\sum_{z \in \Theta_{s f}} \gamma\left(L_{1}^{2}+z^{2}\right)^{-1}\right] \\
& =\int_{\delta_{f f}}^{\infty} \rho \xi \gamma\left(L_{1}^{2}+z^{2}\right)^{-1} d z+\int_{\delta_{0 s f}}^{\delta_{1 s f}} \rho \xi \gamma\left(L_{1}^{2}+z^{2}\right)^{-1} d z \\
& =\frac{\rho \xi \gamma}{L_{1}}\left(\left.\arctan \frac{z}{L_{1}}\right|_{z=\delta_{f f}} ^{z=\infty}+\left.\arctan \frac{z}{L_{1}}\right|_{z=\delta_{0 s f}} ^{z=\delta_{1 s f}}\right) .
\end{aligned}
$$

In the worst case that $\theta \approx 180^{\circ}$, i.e., the antennas have a very low directivity, we have $\delta_{f f}=\delta_{0 s f}=0$ and $\delta_{1 s f}=\infty$, thus

$$
\overline{P_{f}}=\left.\frac{2 \rho \xi \gamma}{L_{1}} \arctan \frac{Z}{L_{1}}\right|_{z=0} ^{z=\infty}=\frac{\pi \rho \xi \gamma}{L_{1}} .
$$

Similarly, for the left radar of the typical car, the aggregated interference $P_{s}$ can be written as

$$
P_{s}=\sum_{z \in \Theta_{\text {Is }}} \gamma\left(L_{1}^{2}+z^{2}\right)^{-1}+\sum_{z \in \Theta_{s s}} \gamma\left(L_{1}^{2}+z^{2}\right)^{-1} .
$$

And the mean value of $P_{s}$ is

$$
\begin{aligned}
\overline{P_{s}} & =\mathbb{E}\left[P_{s}\right] \\
& =\frac{\rho \xi \gamma}{L_{1}}\left(\left.\arctan \frac{z}{L_{1}}\right|_{z=\delta_{0 f s}} ^{z=\delta_{1 f s}}+\left.\arctan \frac{z}{L_{1}}\right|_{z=-\delta_{s s}} ^{z=\delta_{s s}}\right) .
\end{aligned}
$$

In a worst case that $\theta \approx 180^{\circ}$, we have $\delta_{0 f s}=0$ and $\delta_{1 f \mathrm{~s}}=\delta_{\mathrm{ss}}=\infty$, and

$$
\begin{aligned}
\overline{P_{s}} & =\left.\frac{\rho \xi \gamma}{L_{1}} \arctan \frac{z}{L_{1}}\right|_{z=0} ^{z=\infty}+\left.\frac{\rho \xi \gamma}{L_{1}} \arctan \frac{z}{L_{1}}\right|_{z=-\infty} ^{z=\infty} \\
& =\frac{3 \pi \rho \xi \gamma}{2 L_{1}} .
\end{aligned}
$$

Note that while we only consider the simple scenario of a two-lane road, it is straightforward to extend the analysis to the multiple-lane situation.

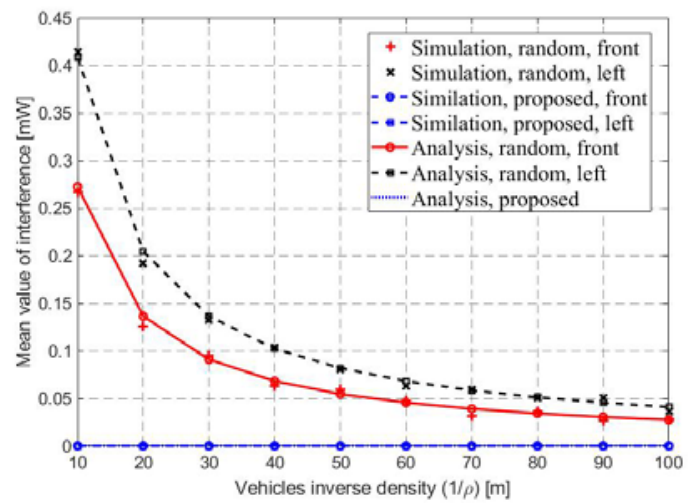

Fig. 4 The interference mean versus vehicle linear intensity.

\subsection{Minimum number of sub-bands required for zero-interference}

Denote $\Gamma$ as a set of radars, where every radar in $\Gamma$ has potential interference with all reminded radars, i.e., the corresponding rows and columns in the potential interference matrix $\boldsymbol{P}$ constitute a sub-matrix, where every elements are nonzero except the main diagonal elements. We named $\Gamma$ as a full-interference set. Note that in $\Gamma$, we have to allocate different orthogonal sub-bands for different radars, otherwise there is interference among the radars in this set. Thus the minimum number of required sub-bands equals to the maximal size of $\Gamma$. Denote the minimum number of required sub-bands as $N$, we have

$$
N=\max (|\Gamma|) .
$$

Generally speaking, $N$ is decided by the locations and the orientations of the vehicles. In Fig. 3 we observe the full-interference set of the straight 
road for the worst case when $\theta \approx 180^{\circ}$. From Fig. 3 we can find that for the straight road, we have $N=2$ if there are two lanes of opposing traffic directions, and $N=4$ if there are four lanes.

\section{SIMULATION RESULTS}

In this section, we present the simulation results for the averaged radar interference and radar ranging success probability, and compare the performance of our proposed sub-bands allocation framework (proposed framework) with the random access scheme (random scheme). The vehicles are deployed over a length of $1000 \mathrm{~m}$ in front of the typical vehicle, and over a length of $1000 \mathrm{~m}$ behind the typical vehicle. We consider the worst case when $\theta \approx 180^{\circ}$. The other simulation parameters can be found in Table I.

TABLE I

\begin{tabular}{ccc}
\multicolumn{3}{c}{ SIMULATION PARAMETERS } \\
\hline Symbol & Value & Explanation \\
\hline $\mathrm{L}_{1}$ & $3.5 \mathrm{~m}$ & Lane width \\
$P_{t}$ & $10 \mathrm{dBm}[25]$ & Transmit power \\
$G$ & $34 \mathrm{dBi}[25]$ & Antenna gain \\
$\sigma_{c}$ & $30 \mathrm{dBsm}[21]$ & Radar cross-section \\
$T h$ & $10 \mathrm{~dB}[21]$ & SINR threshold \\
$f$ & $77 \mathrm{GHz}$ & Center frequency \\
$\theta$ & $180^{\circ}$ & Antenna beam width \\
$\rho$ & Variable cars/m & Linear intensity \\
$T$ & Variable & Number of sub-bands \\
\hline
\end{tabular}

In Fig. 4, we present the average interference versus vehicle linear intensity for the two-lane road. We set $T=2$, and $\rho$ varies from 0.01 to 0.1 . The average interference for both the front radar and the left radar of the typical vehicle is presented. From Fig. 4, first we can see the simulation results match well with the analysis for the random scheme. We can also find that the average interference decreases with the decreasing of vehicles intensity, and the average interference for the left radar is larger than that for the front radar. In addition, for the proposed framework, the simulated average interference for the front radar and the left radar of the typical vehicle is zero, which validates that the proposed framework can reduce interference significantly.

Fig. 5 and Fig. 6 show the average interference versus the number of sub-bands for both two-lane road and four-lane road. The average interference of the front radar and the left radar of the typical vehicle and the normalization total interference for all radars on the road are presented. We set $\rho=0.1$, and vary $T$ from 1 to 6. In both Fig. 5 and Fig. 6, when $T=1$, the average interference is the same for the proposed framework and the random scheme. However, with the increase of $T$, the average interference in the proposed framework decreases much faster than the random scheme. The normalization total interference decreases to zero when $T \geq 2$ in Fig. 5 and when $T \geq 4$ in Fig. 6, which means the proposed framework can achieve zero-interference for all radars with the minimum required number of sub-bands, for both two-lane situation and four-lane situation.

In Fig. 7, we show the radar ranging success probability versus the ranging distance for the left radar of the typical vehicle when the number of lanes is four. We set $\rho=0.01$, and vary $T$ from 1 to 4 .

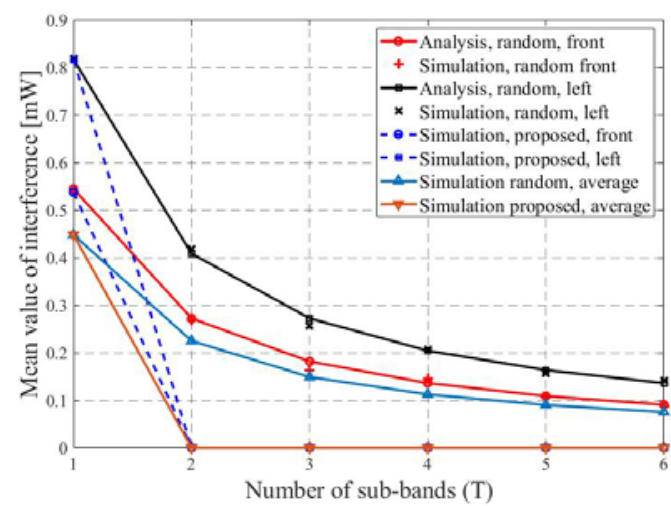

Fig. 5 The average interference versus the number of sub-bands for two-lane road.

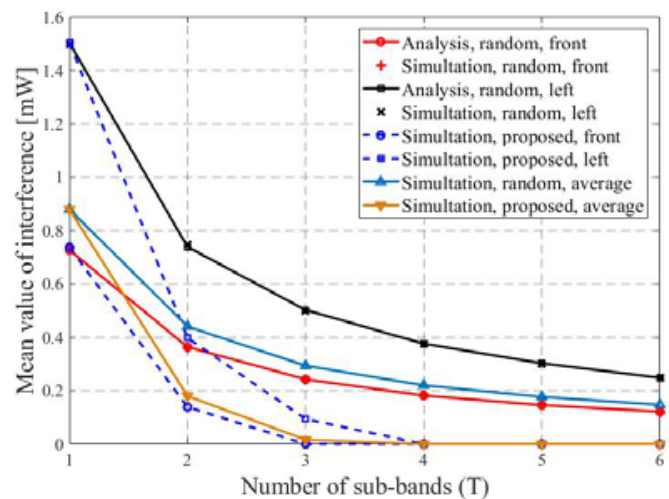

Fig. 6 The average interference versus the number of sub-bands for four-lane road.

Successful ranging and detection require that the SINR is larger than a certain threshold $T h$. For dense traffic conditions, interference limits the performance rather than noise, thus in simulation we calculate the signal to interference ratio (SIR) and count the number of times that it exceeds the threshold to obtain the simulated success probability. From Fig. 7 we can find that for the random scheme, the improvement of the success probability is not obvious with $T$ 
increasing. Moreover, the success probability for the random scheme is lower than 0.9 when the target is more than 10 meters away, and this probability approaches zero when the target range is $30 \mathrm{~m}$. However, the success probability improves notably when $T$ increases by using the proposed framework. When $T=3$, the success probability decreases much slower with target range increasing. And when $T \geq 4$, there is no interference between radars, thus the success probability is only limited by noise. This result gives further insight that the electromagnetic compatibility (EMC) design of the automotive radar may be relaxed with the help of communication systems, e.g., the transmitting power can be increased to improve the detection performance.

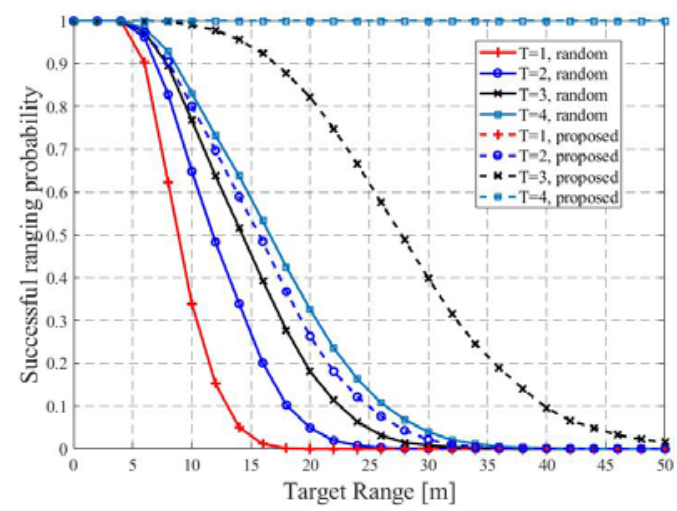

Fig. 7 the radar ranging success probability versus the target distance.

\section{CONCLUSIONS}

In this paper, we proposed a framework that utilizes communication system to allocate orthogonal frequency sub-bands and minimize the automotive radar interference. A greedy algorithm was proposed to resolve the minimization problem based on the location and the parameters of radars. The Poisson point process was used to formulate interfering radars and to calculate the average interference. Moreover, the minimum number of orthogonal frequency sub-bands was also analyzed. The simulation results verified the analytical results, and demonstrated the effectiveness of the proposed framework. It was also validated that the proposed framework could use the minimum number of sub-bands to eliminate interference.

\section{ACKNOWLEDGEMENTS}

This work was supported in part by the European Union's Horizon 2020 research and innovation programme under the Marie Skłodowska-Curie Grant Agreement No. 793345.

\section{REFERENCES}

[1] S. Zhang, J. Chen, F. Lyu, N. Cheng, W. Shi and X. Shen, "Vehicular Communication Networks in the Automated Driving Era," in IEEE Communications Magazine, vol. 56, no. 9, pp. 26-32, SEPTEMBER 2018.

[2] F. J. Martin-Vega, M. C. Aguayo-Torres, G. Gomez, J. T. Entrambasaguas and T. Q. Duong, "Key Technologies, Modeling Approaches, and Challenges for Millimeter-Wave Vehicular Communications," in IEEE Communications Magazine, vol. 56, no. 10, pp. 28-35, OCTOBER 2018.

[3] Y. Li, Y. Cao, H. Qiu, L. Gao, Z. Du and S. Chen, "Big wave of the intelligent connected vehicles," in China Communications, vol. 13, no. Supplement2, pp. 27-41, 2016.

[4] B. Paul, A. R. Chiriyath and D. W. Bliss, "Survey of RF Communications and Sensing Convergence Research," in IEEE Access, vol. 5, pp. 252-270, 2017.

[5] R. Saruthirathanaworakun, J. M. Peha and L. M. Correia, "Opportunistic Sharing Between Rotating Radar and Cellular," in IEEE Journal on Selected Areas in Communications, vol. 30, no. 10, pp. 1900-1910, November 2012.

[6] S. Sodagari, A. Khawar, T. C. Clancy and R. McGwier, "A projection based approach for radar and telecommunication systems coexistence," 2012 IEEE Global Communications Conference (GLOBECOM), Anaheim, CA, 2012, pp. 5010-5014.

[7] F. Liu, C. Masouros, A. Li and T. Ratnarajah, "Robust MIMO Beamforming for Cellular and Radar Coexistence," in IEEE Wireless Communications Letters, vol. 6, no. 3, pp. 374-377, June 2017.

[8] F. Liu, C. Masouros, A. Li, T. Ratnarajah and J. Zhou, "MIMO Radar and Cellular Coexistence: A Power-Efficient Approach Enabled by Interference Exploitation," in IEEE Transactions on Signal Processing, vol. 66, no. 14, pp. 3681-3695, 15 July15, 2018.

[9] L. Zheng, M. Lops and X. Wang, "Adaptive Interference Removal for Uncoordinated Radar/Communication Coexistence," in IEEE Journal of Selected Topics in Signal Processing, vol. 12, no. 1, pp. 45-60, Feb. 2018.

[10] P. Kumari, J. Choi, N. González-Prelcic and R. W. Heath, "IEEE 802.11ad-Based Radar: An Approach to Joint Vehicular Communication-Radar System," in IEEE Transactions on Vehicular Technology, vol. 67, no. 4, pp. 3012-3027, April 2018.

[11] J. A. Zhang, A. Cantoni, X. Huang, Y. J. Guo and R. W. Heath, "Framework for an Innovative Perceptive Mobile Network Using Joint Communication and Sensing," 2017 IEEE 85th Vehicular Technology Conference (VTC Spring), Sydney, NSW, 2017, pp. 1-5.

[12] J. A. Zhang, X. Huang, Y. J. Guo and M. L. Rahman, "Signal stripping based sensing parameter estimation in perceptive mobile networks," 2017 IEEE-APS Topical Conference on Antennas and Propagation in Wireless Communications (APWC), Verona, 2017, pp. 67-70.

[13] F. Liu, L. Zhou, C. Masouros, A. Li, W. Luo and A. Petropulu, "Toward Dual-functional Radar-Communication Systems: Optimal Waveform Design," in IEEE Transactions on Signal Processing, vol. 66, no. 16, pp. 4264-4279, 15 Aug.15, 2018.

[14] N. González-Prelcic, R. Méndez-Rial and R. W. Heath, "Radar aided beam alignment in MmWave V2I communications supporting antenna diversity," 2016 Information Theory and Applications Workshop (ITA), La Jolla, CA, 2016, pp. 1-7.

[15] M. Kunert, "The EU project MOSARIM: A general overview of project objectives and conducted work," 2012 9th European Radar Conference, Amsterdam, 2012, pp. 1-5.

[16] G. M. Brooker, "Mutual Interference of Millimeter-Wave Radar Systems," in IEEE Transactions on Electromagnetic Compatibility, vol. 49, no. 1, pp. 170-181, Feb. 2007.

[17] T. Luo, C. E. Wu and Y. E. Chen, "A 77-GHz CMOS Automotive Radar Transceiver With Anti-Interference Function," in IEEE Transactions on Circuits and Systems I: Regular Papers, vol. 60, no. 12, pp. 3247-3255, Dec. 2013.

[18] T. Luo, C. E. Wu and Y. E. Chen, "A 77-GHz CMOS FMCW Frequency Synthesizer With Reconfigurable Chirps," in IEEE 
Transactions on Microwave Theory and Techniques, vol. 61, no. 7, pp. 2641-2647, July 2013.

[19] A. Al-Hourani, R. J. Evans, S. Kandeepan, B. Moran and H. Eltom, "Stochastic Geometry Methods for Modeling Automotive Radar Interference," in IEEE Transactions on Intelligent Transportation Systems, vol. 19, no. 2, pp. 333-344, Feb. 2018.

[20] M. I. Skolnik, Ed., Radar Handbook, 3rd ed. New York, NY, USA: McGraw-Hill, 2008.

[21] J. Hasch, E. Topak, R. Schnabel, T. Zwick, R. Weigel and C. Waldschmidt, "Millimeter-Wave Technology for Automotive Radar Sensors in the $77 \mathrm{GHz}$ Frequency Band," in IEEE Transactions on Microwave Theory and Techniques, vol. 60, no. 3, pp. 845-860, March 2012.

[22] S. M. Patole, M. Torlak, D. Wang and M. Ali, "Automotive radars: A review of signal processing techniques," in IEEE Signal Processing Magazine, vol. 34, no. 2, pp. 22-35, March 2017.

[23] C. Shao, S. Leng, Y. Zhang, A. Vinel, and M. Jonsson, "Performance analysis of connectivity probability and connectivity-aware MAC protocol design for platoon-based VANETs," IEEE Transactions on Vehicular Technology, vol. 64, no. 12, pp. 5596-5609, Dec 2015.

[24] M. Haenggi, Stochastic Geometry for Wireless Networks. Cambridge, U.K.: Cambridge Univ. Press, 2012.

[25] L. H. Eriksson and B. As, "A high performance automotive radar for automatic AICC," in IEEE Aerospace and Electronic Systems Magazine, vol. 10, no. 12, pp. 13-, Dec. 1995.

[26] L. Eriksson and S. Broden, "High performance automotive radar,” in Microw. J., vol. 49, pp. 24-38, Oct. 1996.

\section{BIOGRAPHIES}

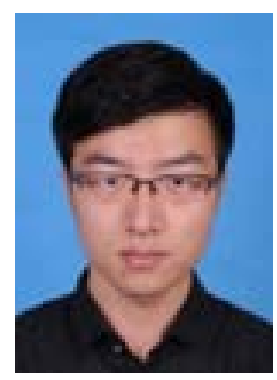

Jingxuan Huang, received the B.S. degree in electronics engineering from the Beijing Institute of Technology (BIT), Beijing, China, in 2016. He is currently pursuing the Ph.D. degree with the Research Institute of Communication Technology, BIT. His research interests include channel coding and modulation, mobile communication, and vehicular communications. Email: jxhbit@gmail.com

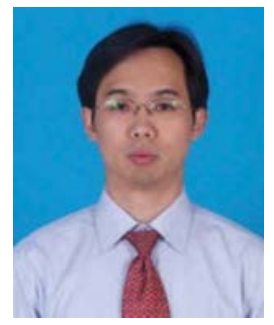

Zesong Fei, received the Ph.D. degree in electronic engineering from the Beijing Institute of Technology (BIT), in 2004. He is currently a Professor with BIT and currently with the Research Institute of Communication Technology, BIT, where he is involved in the design of the next generation high-speed wireless communication. His research interests include wireless communications and multimedia signal processing. He is the Chief Investigator of the National Natural Science Foundation of China. He is the Senior Member of the Chinese Institute of Electronics and the China Institute of Communications. Email: feizesong@bit.edu.cn

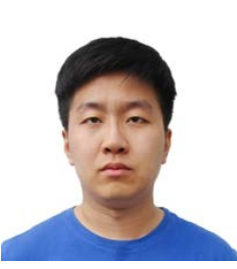

Tianxiong Wang, is currently pursuing the B.S. degree in electronic science and technology from the Beijing Institute of Technology (BIT), Beijing, China, from 2015. His research interests include channel modelling, vehicular networks, OFDM, and convex optimization. Email: wangtianxiong @bit.edu.cn

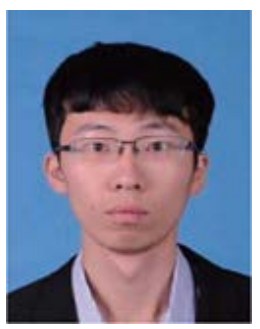

Xinyi Wang, received the B.S. degree in electronics engineering from the Beijing Institute of Technology (BIT), Beijing, China, in 2017. He is currently pursuing the Ph.D. degree with the Research Institute of Communication Technology, BIT. His research interests include channel coding, mobile communication, and joint radar and communications. Email: bit_wangxy@163.com

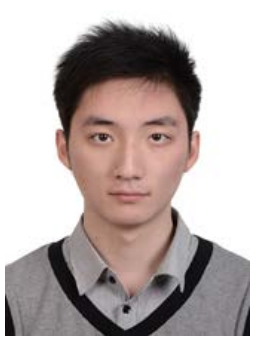

Fan Liu, received the Bachelor degree in information engineering and the Ph.D. degree in electronics science and technology in 2013 and 2018, respectively, all from Beijing Institute of Technology. From 2016 to 2018, he was with the Department of Electrical and Electronic Engineering, University College London, London, U.K., as a visiting student, where he is currently a Postdoctoral Research Associate. His research interests include wireless communication, MIMO radar signal processing and convex optimization. He received the Marie Curie Individual Fellowship in 2018, and has been recognized as an Exemplary Reviewer for the IEEE Transactions on Wireless Communications, the IEEE Transactions on Communications and the IEEE Communications 


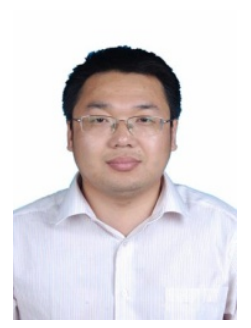

Haijun Zhou, received the B.S. degree in electronics engineering from the Chongqing University of Posts and Telecommunications, Chongqing, China, in 2003. He is currently pursuing the Ph.D. degree with the Research Institute of Communication Technology, BIT. His research interests include Cellular V2X (C-V2X) and mobile communication. Email: zhouhaijun@catt.cn

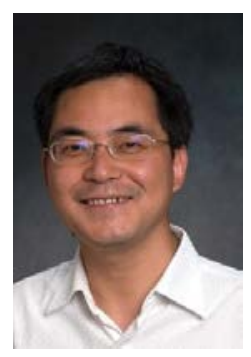

J. Andrew Zhang, received the B.Sc. degree from Xi'an JiaoTong University, China, in 1996, the M.Sc. degree from Nanjing University of Posts and Telecommunications, China, in 1999, and the Ph.D. degree from the Australian National University, in 2004.

Currently, Dr. Zhang is an Associate Professor in the School of Electrical and Data Engineering, University of Technology Sydney, Australia. He was a researcher with Data61, CSIRO, Australia from 2010 to 2016, the Networked Systems, NICTA, Australia from 2004 to 2010, and ZTE Corp., Nanjing, China from 1999 to 2001. Dr. Zhang's research interests are in the area of signal processing for wireless communications and sensing, and autonomous vehicular networks. He has published more than 130 papers in leading international Journals and conference proceedings, and has won 4 best paper awards for his work. He is a recipient of CSIRO Chairman's Medal and the Australian Engineering Innovation Award in 2012 for exceptional research achievements in multi-gigabit wireless communications. Email: Andrew.Zhang@uts.edu.au

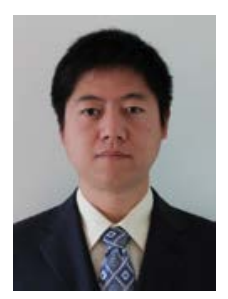

Guohua Wei, received the B.Sc. degree from Xi'an JiaoTong University, China, in 1996, the M.Sc. degree from Nanjing University of Posts and Telecommunications, China, in 1999, and the Ph.D. degree from the Australian National University, in 2004. Email: ghwei@bit.edu.cn 